\title{
Análisis bibliométrico sobre la producción científica archivística en la Red de Revistas Científicas de América Latina y el Caribe (Redalyc) durante el período 2001-2011
}

\author{
Leomar José Montilla Peña \\ Universidad Politécnica Territorial Andrés Eloy Blanco - Lara, Venezuela
}

ARTÍCULO / ARTICLE

\begin{abstract}
Resumen
Objetivo. Analiza la producción científica archivística registrada en la hemeroteca de acceso libre Red de Revistas Científicas de América Latina y el Caribe (Redalyc).

Método. Se aplicó un estudio bibliométrico con un muestreo que incluyó un análisis estadístico longitudinal desde el año 2001 hasta 2011. Se desarrollaron búsquedas avanzadas por palabras clave relacionadas a la archivística.

Resultados. Se identificó que el año de mayor productividad fue el 2011 con un total de 19 publicaciones, siendo Biblios la revista que más ha publicado sobre archivística con 22 artículos, encontrándose que el $68,38 \%$ de la producción autoral son firmados por un solo autor.

Conclusiones. En el decenio estudiado se registraron en Redalyc un total de 117 publicaciones referidas al área, existiendo un crecimiento gradual en los últimos años en la productividad científica archivística. Se identificó que los temas más comunes son: archivos como medio de acceso a la información, fuentes documentales.
\end{abstract}

Palabras clave

Archivística ; Producción científica ; Estudios métricos de la información ; Bibliometría ; Redalyc

Bibliometric analysis about archival scientific production in the Network of Scientific Journals of Latin America and the Caribbean (Redalyc) during the period 2001- 2011

\section{Abstract}

Objective. Analyze the archival scientific production registered in the Network of Scientific Journals of Latin America and the Caribbean (Redalyc).

Method. Applied a bibliometric study with a sample that included a longitudinal statistical analysis from 2001 to 2011 . Searches advanced by key words related to the archives have been developed.

Results. The year of increased productivity was the 2011 with a total of 19 publications, being Biblios the journal with more published articles, finding that $68,38 \%$ of the authorial production are signed by a single author.

Conclusions. A total of 117 publications are refered to $\mathrm{s}$ area and were recorded in Redalyc, with a gradual growth in the last years in the archival scientific productivity. The most common identified topics are: archives for to access to information, and documentary sources.

Keywords

Archives ; Scientifc production ; Metric information studies; Bibliometrics ; Redalyc 


\section{Introducción}

Dada la importancia que tienen los estudios bibliométricos para evaluar el surgimiento y desarrollo de las actividades propias del conocimiento y conocer la actividad científica de sus investigadores e instituciones se planteó como objetivo analizar la producción científica archivística a partir de un estudio bibliométrico en la hemeroteca en línea y de acceso libre Red de Revistas Científicas de América Latina y el Caribe (Redalyc) durante el periodo 2001 hasta el 2011.

Este planteamiento se traza en un estudio Bibliométrico debido a la estrecha relación existente entre la matemática y la estadística con el fin de evaluar la productividad científica y lograr la matematización de los resultados para la toma de decisiones, la cual ha sido ampliamente definida por Tague-Sutcliffe (1994) según el cual la Bibliometría es:

(...) el estudio de aspectos cuantitativos de producción y diseminación y uso de información registrada, a cuyo efecto desarrolla modelos y medidas matemáticos, que sirven para hacer pronósticos y tomar decisiones en torno a tales procesos (p. 2).

A su vez, Spinak (1996) basado en la definición de Pritchard (1969) precisa a la bibliometría como:

La aplicación de las matemáticas y los métodos estadísticos para analizar el curso de la comunicación escrita y el curso de una disciplina. Dicho de otra manera, es la aplicación de tratamientos cuantitativos a las propiedades del discurso escrito y los comportamientos típicos de éste (p. 34).

Más recientemente, Rubio (2004) amplia este modelo de estudio métrico bajo dos perspectivas:

La Bibliometría descriptiva trata de aspectos puramente cuantitativos, como distribución geográfica, documental, temática y su productividad. Mientras que la Bibliometría evaluativa complementa la primera con estudios de evaluación de la actividad científica mediante la aplicación de técnicas estadísticas y programas informáticos de mayor complejidad.

La matematización de los resultados obtenidos en esta investigación se da a través de la formulación lógica y ordenada de los hechos, el análisis de la situación, un adecuado uso del lenguaje, la búsqueda de analogías entre ésta y otras situaciones por medio de los indicadores, con base en esto Chaviano (2004) sostiene que:

Los indicadores revisten especial importancia en los estudios métricos. Cada estudio utiliza una serie de indicadores particulares. De su selección depende, en gran medida, la calidad y el impacto de la investigación final. Ellos proporcionan información cuantitativa y objetiva sobre los resultados el proceso de investigación, su volumen, evolución, visibilidad, estructura, etcétera ( $p$. 1).

La investigación se encuentra dentro de la metodología cuantitativa, por otra parte, el estudio se apoya en una investigación documental, que de acuerdo a Arias (2006):

Es un proceso basado en la búsqueda, recuperación, análisis, crítica e interpretación de datos secundarios, es decir, los obtenidos y registrados por otros investigadores en fuentes documentales: impresas, audiovisuales o electrónica (p. 27).

Por ello, la Bibliometría reviste para efecto de este estudio un papel importante por ser una de los estudios metrícos de la información más sólidas dentro del mundo informacional resultando un método confiable y universal para medir la productividad en esta área de conocimiento específico y así obtener resultados fiables para la toma de decisiones.

La producción científica archivística ha generado diversos tipos de documentos y como ciencia ha evolucionado en su acepción. Para entender a cabalidad la ocupación archivística y comprender el fundamento del análisis bibliométrico que se presenta en este artículo, se hace necesario abordar algunas definiciones de esta ciencia, entre

ellas encontramos la del diccionario de terminología archivística del Consejo Internacional de Archivos (1988), la archivística es el estudio teórico y práctico de los principios, procedimientos y problemas concernientes a las funciones de los archivos. 
Referente a la archivística, Heredia (1991) en su monografía señala que:

Es la ciencia de los archivos, no de los documentos, aunque en última instancia éstos sean el producto integrante de aquéllos. Como tal se ocupará de la creación, historia, organización y servicio de los mismos a la administración y a la historia, en definitiva a la sociedad (p. 29).

Cruz (1994) en su obra considera que la archivística es:

(...) una ciencia emergente. Es ciencia por cuanto posee un objeto, los archivos en su doble consideración: los fondos documentales y su entorno; posee, además, un método, compuesto por un conjunto de principios teóricos y procedimientos prácticos, cuya evolución constante la perfilan con mayor nitidez día a día. Y un fin: hacer recuperable la información documental para su uso ( $p$. 64).

Fuster (2001) afirma en su artículo que la archivística es la:

(...) ciencia que se ocupa de los archivos en sus aspectos teóricos y prácticos, estableciendo principios inalterable y estudiando técnicas adecuadas a la gestión de documentos, administración y tratamiento técnico de archivo, así como la función jurídica, administrativas y científicas de los mismos, desde un punto de vista archivístico o de ciencias o técnicas diversas, y su relación con las entidades productoras de los conjuntos orgánicos de documentos, a fin de manejar y hacer accesible la información de los fondos documentales (p.17).

Cabe manifestar, que a la luz del análisis de la literatura concerniente a la ciencia archivística, esta, ha avanzado significativamente, observándose durante varios espacios históricos diversos enfoques ligados a su desarrollo con el propósito de organizar y conservar la memoria colectiva de las instituciones y de los individuos como hecho fehaciente de las funciones y actividades que cumplen cada uno de ellos.

Existen numerosos ejemplos de estudios realizados con análisis bibliométrico sobre la producción científica en diferentes áreas. Referente a la productividad científica archivística Dorado y Martínez (2010), realizaron un estudio sobre Representatividad de las fuentes de información sobre Archivística en las Bibliotecas Especializadas en la Ciudad de la Habana, basado en el método de evaluación de la productividad científica en siete bibliotecas especializadas, donde ponen a disposición los fenómenos encontrados en su investigación.

La anterior investigación apunta a la productividad científica archivística registrada en bibliotecas especializadas, pero la productividad archivística en hemeroteca de libre acceso en línea, no lo hace. Por tal razón se orientó este análisis bibliométrico sobre la productividad científica relativo a la archivística que se encuentra registrada Redalyc.

\section{Materiales y métodos}

El estudio se realizó en la hemeroteca en línea Red de Revistas Científicas de América Latina y el Caribe (Redalyc), hecho motivado por ser de acceso libre y por difundir la producción científica editada en y sobre Iberoamérica en diferentes áreas del conocimiento, lo que permite realizar un análisis sobre productividad y colaboración desde este contexto.

La variable estudiada fue la producción científica archivística a través de un muestreo estadístico longitudinal comprendido desde el año 2001 hasta 2011. Para la recolección de los artículos se utilizó la base de dato, se aplicó el modelo búsqueda avanzada manejando varias palabras clave y para optimizar el proceso se seleccionaron varias palabras todas relacionadas con el término "Archivística".

Para ello, se tomaron las siguientes acciones: conocer los aspectos conceptuales sobre la archivística y la bibliometría, caracterizar a Redalyc, definir los indicadores bibliométricos que fueron utilizados para el análisis de la producción científica sobre archivística, aplicar los indicadores bibliométricos seleccionados y analizar los datos obtenidos a partir del análisis bibliométrico.

El conjunto de información analizada proviene de 117 artículos, los cuales son firmados por 159 autores pertenecientes a 16 países y registrados en 50 revistas, todas las publicaciones son consideradas del área archivística y recolectadas durante los meses de julio y agosto de 2012 desde la perspectiva hermenéutica y dogmática con el propósito de evitar posibles errores, para ello se procedió a un arduo trabajo de búsqueda, 
ordenación y clasificación de acuerdo a las preguntas formuladas en la investigación y los indicadores seleccionados.

El procesamiento digital de la información se realizó con el paquete computacional Microsoft Excel 2007, permitiendo introducir los datos con el fin de lograr un análisis efectivo lográndose obtener tres (3) tablas y cinco (5) figuras. Para el análisis de redes sociales fue empleado el UCINET, obteniendo tres (3) figuras, la primera para establecer la red de colaboración autoral, la segunda para determinar la colaboración de los autores en más de una revista y la última para determinar la concurrencia de las palabras clave, todo ello con la intención de mostrar un análisis descriptivo referente a la producción científica archivística registrada en Redalyc.

Finalmente, el análisis del estudio Bibliométrico se fundamentó en cada nivel productivo de acuerdo a los indicadores de productividad autoral, productividad de artículos por revistas, colaboración en más de una revista, capacidad idiomática, productividad de artículos por año, productividad de artículos por país, categorías temáticas, productividad institucional y colaboración autoral.

\section{Resultados y discusión}

Se presentan los resultados encontrados en la investigación, en la tabla $N^{\circ} 1$, se puede observar que de 159 autores que realizaron publicaciones en el área archivística y que se encuentran registrados en Redalyc durante el período 2001-2011, 12 autores predominan con mayor productividad de los cuales 2 de ellos presentan 3 publicaciones científicas cada uno, 10 autores realizaron 2 publicaciones y un total de 149 autores publicaron solamente un artículo, los autores más productivos son el Doctor Caldera Serrano Jorge Docente en la universidad de Extremadura y la Doctora Blanca Rodríguez Bravo Docente en la Universidad de León.

\begin{tabular}{|c|c|}
\hline \multicolumn{2}{|l|}{ PRODUCTIVIDAD AUTORAL } \\
\hline AUTOR & $\mathrm{N}^{\circ}$ de Artículos \\
\hline Caldera Serrano Jorge & 3 \\
\hline Rodríguez Bravo Blanca & 3 \\
\hline Chaín Navarro Celia & 2 \\
\hline García Pérez María Sandra & 2 \\
\hline Marin Agudelo Sebastian Alejandro & 2 \\
\hline Navarro Bonilla Diego & 2 \\
\hline Portillo Lisbeth & 2 \\
\hline Rivas Fernández José Bernal & 2 \\
\hline Sánchez Gómez Arcángel Eduardo & 2 \\
\hline Vivas Moreno Agustín & 2 \\
\hline Yaén García Luís Fernando & 2 \\
\hline Zapata Cárdenas Carlos Alberto & 2 \\
\hline 149 Autores & 1 \\
\hline
\end{tabular}

Tabla 1 - Distribución de autores según el número de publicaciones en el área archivística en Redalyc 20012011

En los datos analizados y que se muestran en la tabla $N^{\circ} 2$, se visualiza que las publicaciones con 3 autores tiene un $5,98 \%$, las de 4 y 5 autores son los de menor porcentaje significativo con un 3,42\% mientras los de una autoría son excesivamente alto con un $68,38 \%$, siguiéndole las publicaciones con dos autoría $22,22 \%$, encontrando el mayor protagonismo entre los de uno y dos autores con $90,6 \%$ en comparación con los otros. 


\begin{tabular}{ccc}
\hline $\mathbf{N}^{\circ}$ de Autores & $\mathbf{N}^{\circ}$ de Artículos & $\begin{array}{c}\% \\
\text { Significativo }\end{array}$ \\
\hline 1 & 80 & 68,38 \\
2 & 26 & 22,22 \\
3 & 7 & 5,98 \\
4 & 2 & 1,71 \\
5 & 2 & 1,71 \\
\cline { 2 - 3 } & 117 & 100
\end{tabular}

Tabla 2 - Distribución de contribuciones de autoría según el número de publicaciones en el área archivística en Redalyc 2001-2011

El análisis social de colaboración autoral se observa entre los nodos rojos en la figura № 1, de los 117 artículos analizados para esta investigación 26 de ellos fueron publicados por dos 2 autores, siete 7 artículos por 3 autores y 2 artículos por 4 y 5 autores, lo que deja ver que la comunidad de investigadores científicos se caracteriza entre otras cosas por trabajar preferiblemente de manera individual.

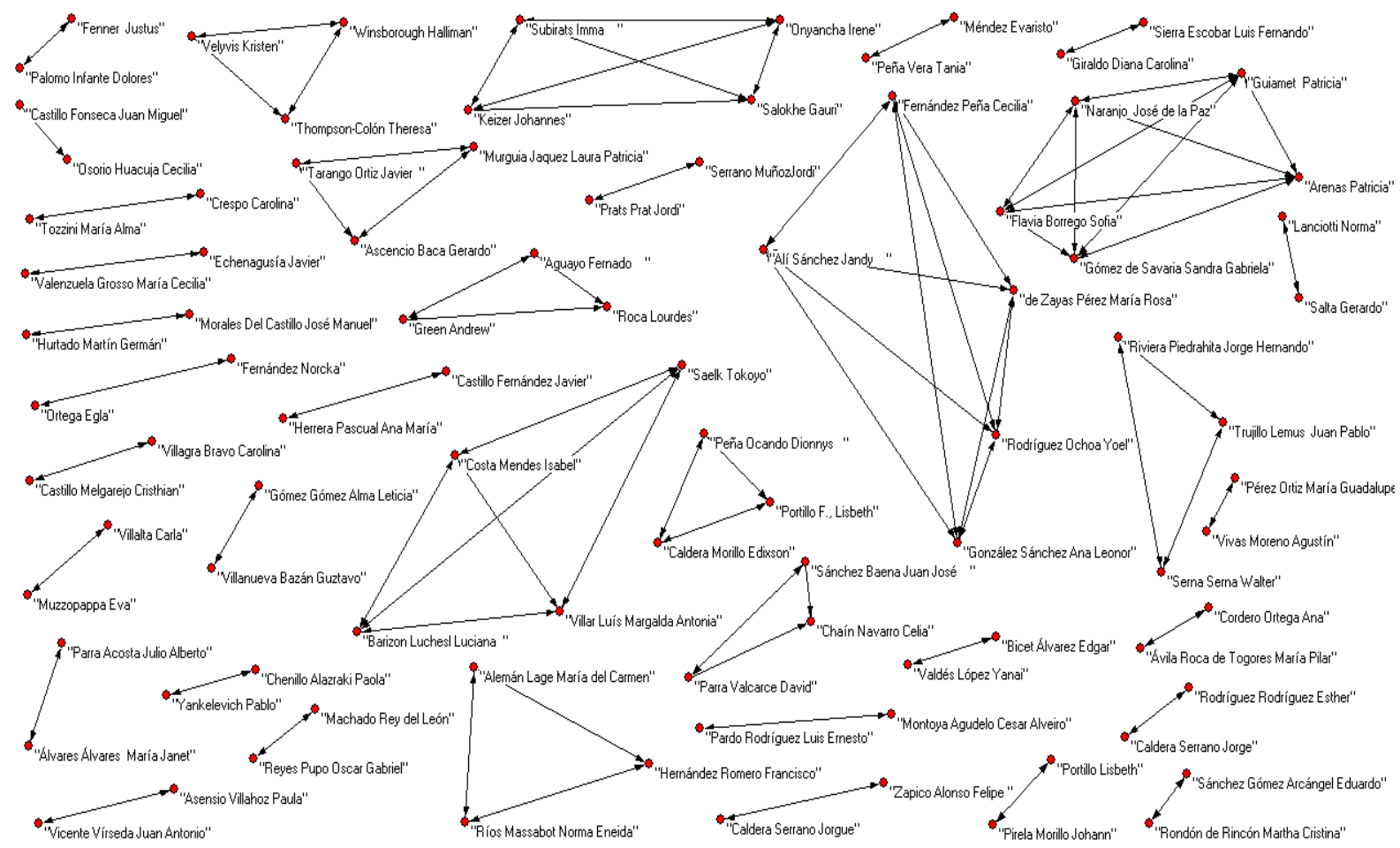

Figura 1 - Red de Colaboración Autoral

La producción científica Archivística registrada en Redalyc de acuerdo al período estudiado fue publicada en 50 revistas, se visualiza en figura $\mathrm{N}^{\circ} 2$ las 15 revistas que presentaron mayor productividad, entre las cuales se encuentran en primer lugar la Revista Biblios con 22 artículos, en segundo Anales de Documentación con 15 publicaciones, en tercero Revista Colombiana de Antropología con 8 y le siguen Desacatos con 6, Revista 
Interamerica de Bibliotecología con 5, Ciencias de la Información y Enl@ce con 4 publicaciones, Aisthesis y Biblioteca Universitaria con 3 artículos y Antíteses, Ciencias Holguín, Diálogos Revista Electrónica de Historia, Historia Crítica, Iconos Revista de Ciencias Sociales y Nómadas 2 publicaciones cada una, mientras las 35 revistas restante se mantienen con una sola publicación.

\section{Productividad de Artículo por Revista}

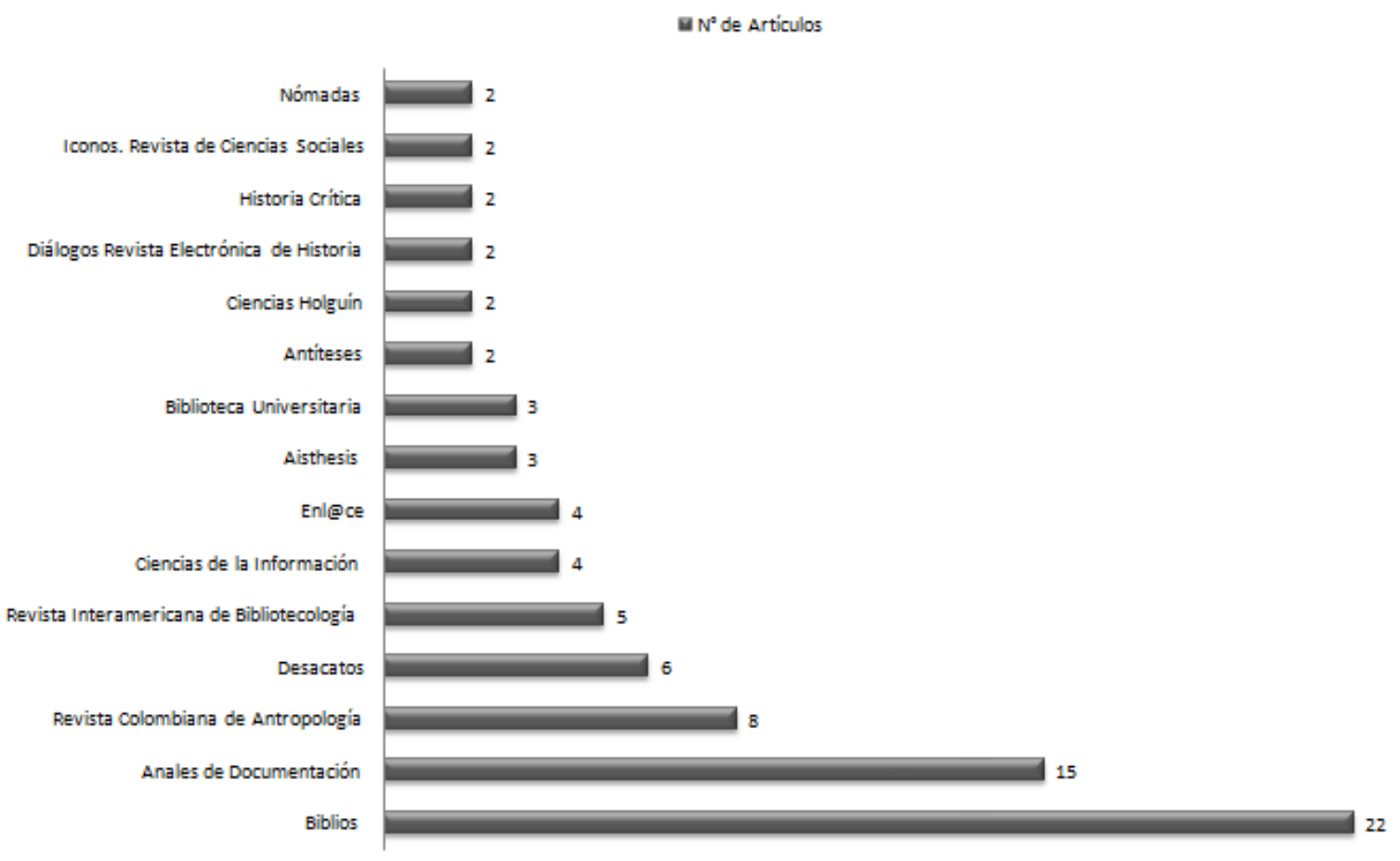

Figura 2 - Revistas con más artículos publicados en Redalyc 2001-2011

El análisis social de colaboración en más de una revista se visualiza en la figura № 3, de los 159 autores que produjeron artículos durante el 2001-2011 en las cincuentas revista obtenidas en Redalyc durante la investigación, solo 6 autores por lo menos han participado en dos revistas representándose en los nodos rojos y la que más han compartido autores son Anales de la Documentación y Biblios con 4 autores de los 6 encontrados.

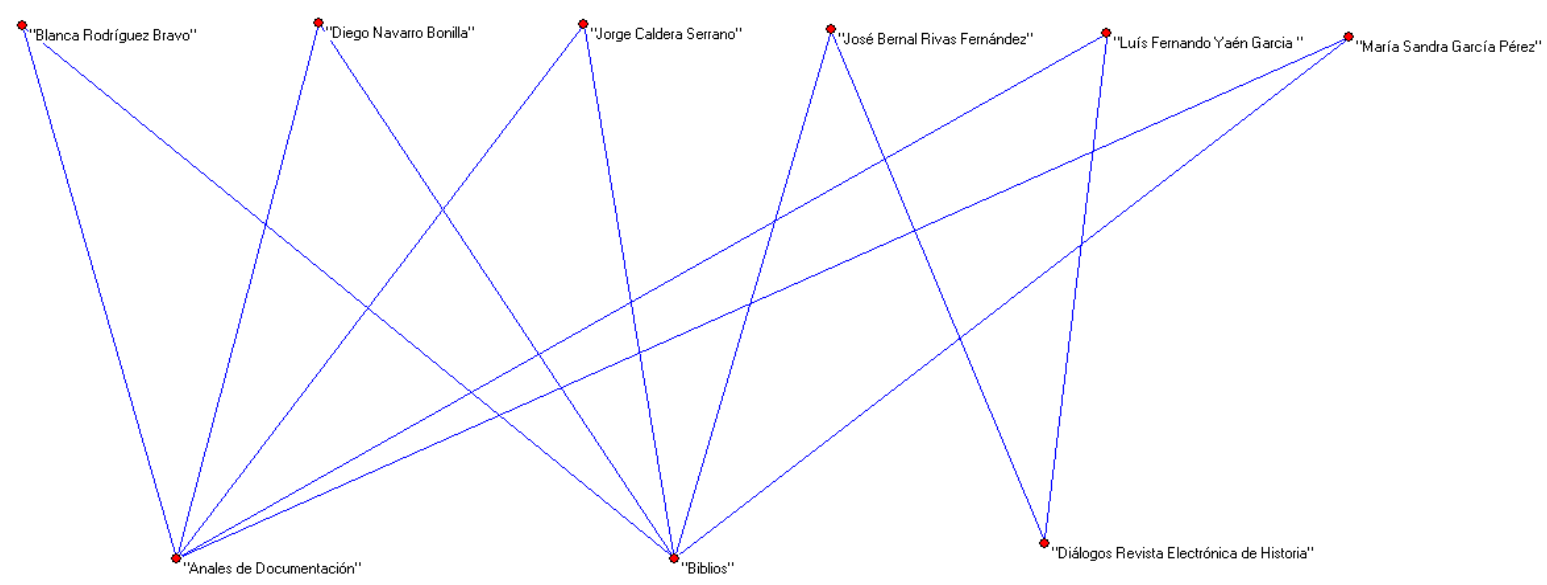

Figura 3 - Red de Colaboración en más de una Revista 
En relación al idioma más productivo en las publicaciones científicas Archivísticas que se encuentran en Redalyc se pudo observar que de los 117 artículos analizados solo se presentan 3 idiomas diferentes, sobresaliendo el español con la mayoría de las publicaciones con un total de 113 como se muestra claramente en la figura $\mathrm{N}^{\circ} 4$, en ingles solo se realizaron 3 publicaciones y en portugués una.

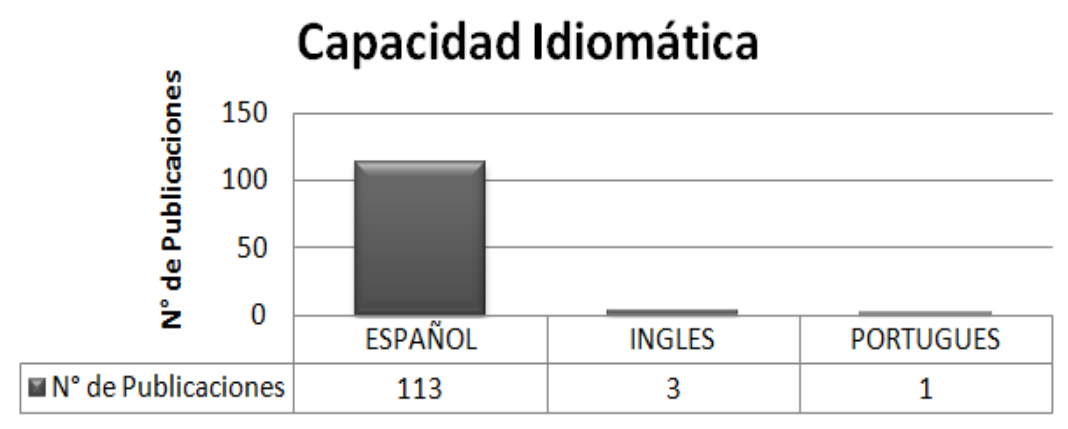

Figura 4 - Productividad Idiomática en Redalyc 2001-2011

En la figura $N^{\circ} 5$ muestra los 10 años de productividad científica Archivística registrados en Redalyc de acuerdo con análisis estadístico longitudinal establecido en el estudio. El análisis evidencia que durante el transcurso del año 2001 se publicaron 4 artículos, durante el 2002 y 2003 se incrementa y se mantienen con 11 artículos, en el 2004 se origina una declinación contundente con la diferencia de 8 artículo menos del margen continuo durante los dos años anteriores, en el 2005 se da un aumento gradual con la publicación de 9 artículos, declinando nuevamente la producción científica archivística durante el 2006 con la producción de 5 artículos, en el 2007 y 2008 se remonta nuevamente de forma progresiva con la producción de 11 y 17 publicaciones declinando reiteradamente para el 2009 con la producción de 12 artículo, para el 2010 se acrecienta 15 artículo y el 2011es el año con mayor productividad de publicaciones con un total de 19 publicaciones.

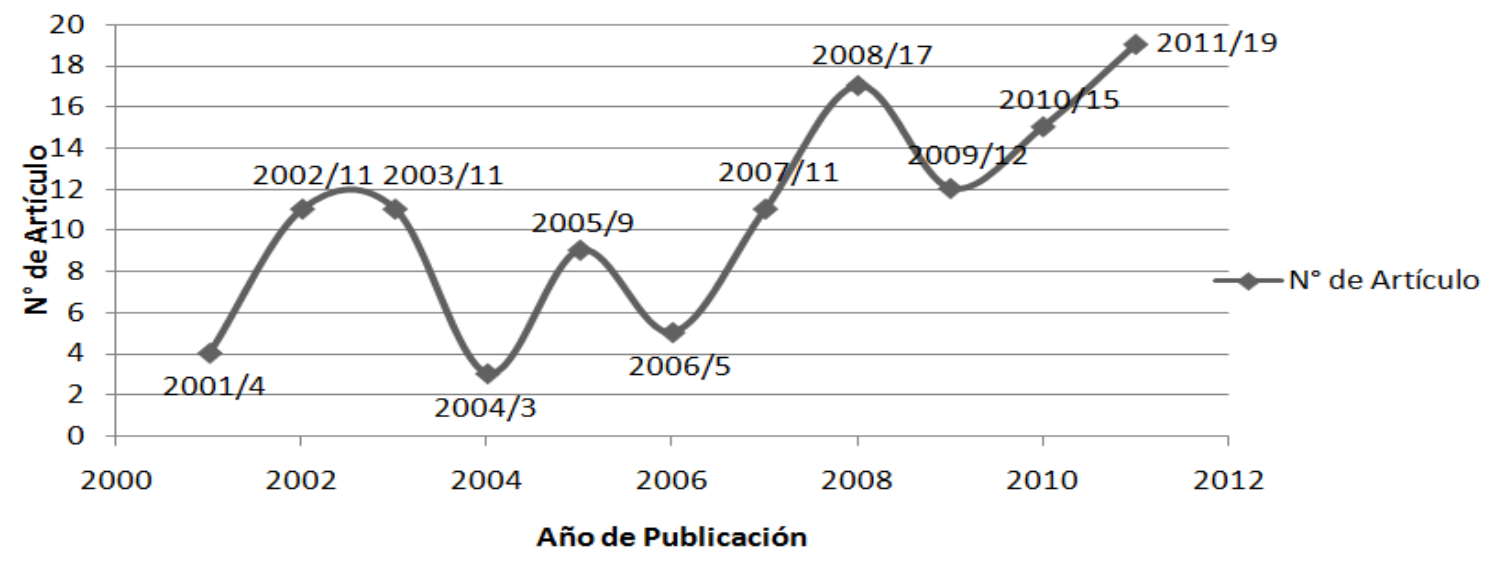

Figura 5 - Productividad de artículos por año en Redalyc 2001-2011

Los resultados obtenidos en relación a la productividad de artículos por países va de acuerdo a la nacionalidad de los autores y al periodo estudiado, se muestra en la figura $\mathrm{N}^{\circ} 6$ donde se visualizan un total de 16 países que generaron publicaciones además de apreciar la colaboración entre 2 países con una publicación, entre los cuales se destaca; España como el país con más publicaciones, éste presenta un total de 30 artículos publicados, seguidamente se muestra Colombia con 15 publicaciones, Argentina y México con 12, Venezuela con 10, Cuba con 8, Chile con 7, Brasil con 6, Costa Rica con 4, Estados Unidos y Uruguay con 3, Perú con 2, Ecuador, Italia, Portugal y Puerto Rico con una publicación, mientras que Argentina y Cuba realizan 1 publicación. 


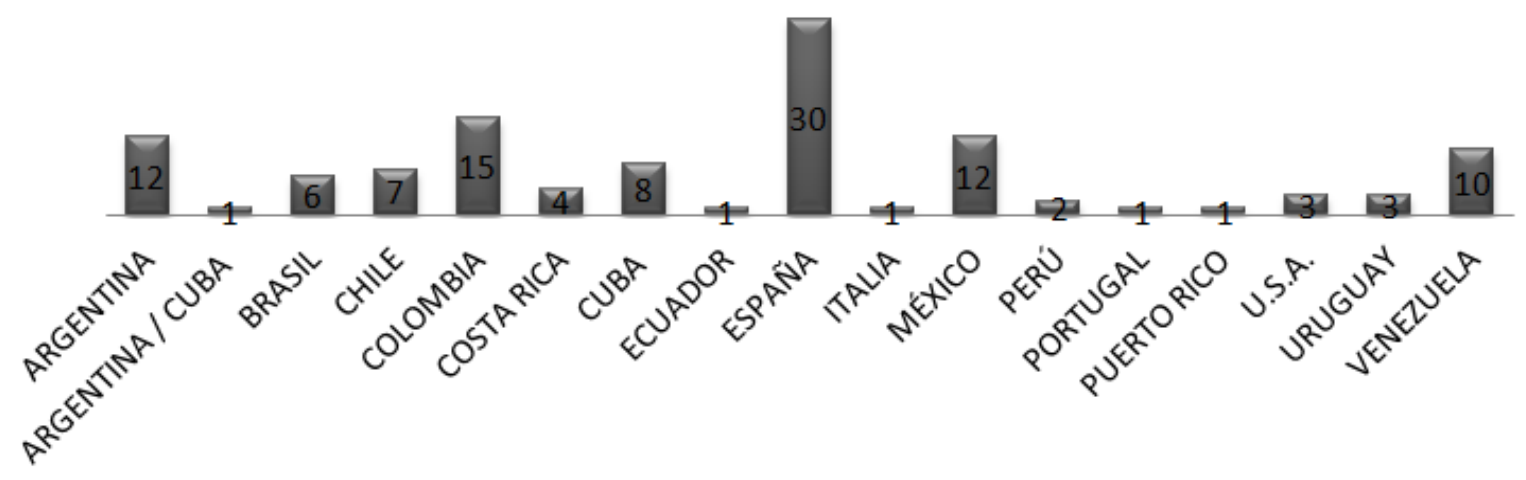

Figura 6 - Productividad de artículos por país en Redalyc 2001-2011

De los 117 artículos analizados se extrajeron 447 palabras clave las cuales se clasificaron y ordenaron obteniendo entre estas las de mayor frecuencia y que se muestran en la figura $N^{\circ} 7$, destacándose en primer lugar la palabra Archivos con un total de 23 repetición, le sigue Archivo con 13 repeticiones, Archivística con 12, Etnografía con 8, Historia y Memoria con 5 repeticiones cada una, Argentina, Gestión de Información, Gestión Documental, Legislación Archivística, Televisión, Teoría Archivística y Web Semántica con 4 repeticiones cada una, 6 palabras claves con 3 repeticiones cada una, 33 palabras clave con 2 repeticiones cada una y 269 descriptores temáticos con una sola frecuencia. Hay que exaltar que la mayor frecuencia del descriptor Archivos se encuentren en los artículos publicados por Biblios con un total de 7 repeticiones.

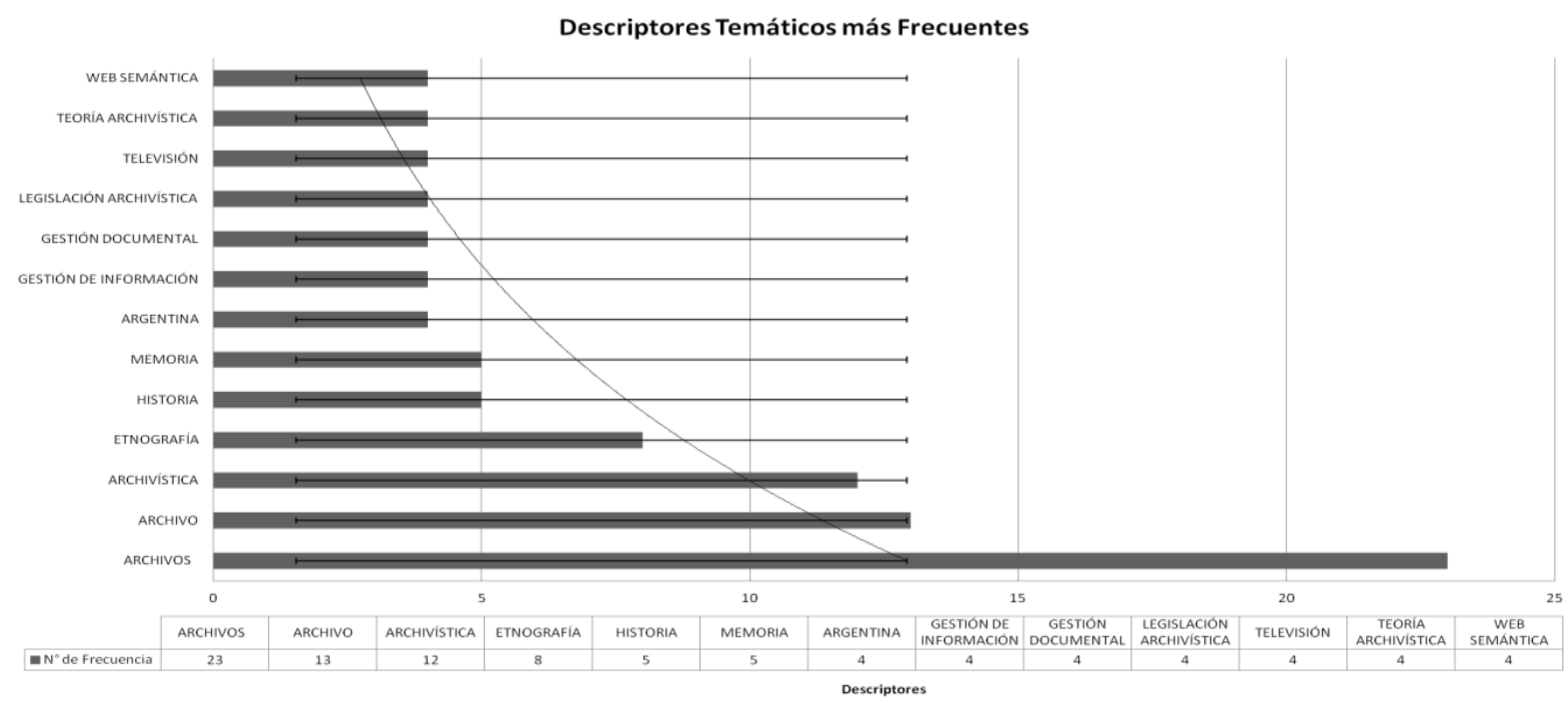

Figura 7 - Temáticas más Frecuentes en Redalyc (2001-2011)

La Figura $N^{\circ} 8$ presenta una dimensión de las concurrencia encontradas en las palabras clave o descriptores temáticos con mayor frecuencia encontrados en los 117 artículos relacionados a la producción científica archivística en Redalyc durante el 2001 - 2011. En primera instancia se aprecia que las palabras con mayor frecuencia se encuentran cerca del núcleo " $\mathrm{N}^{\circ}$ de Presencia" observándose que los nodos rojos con menor frecuencia se van distanciando. 


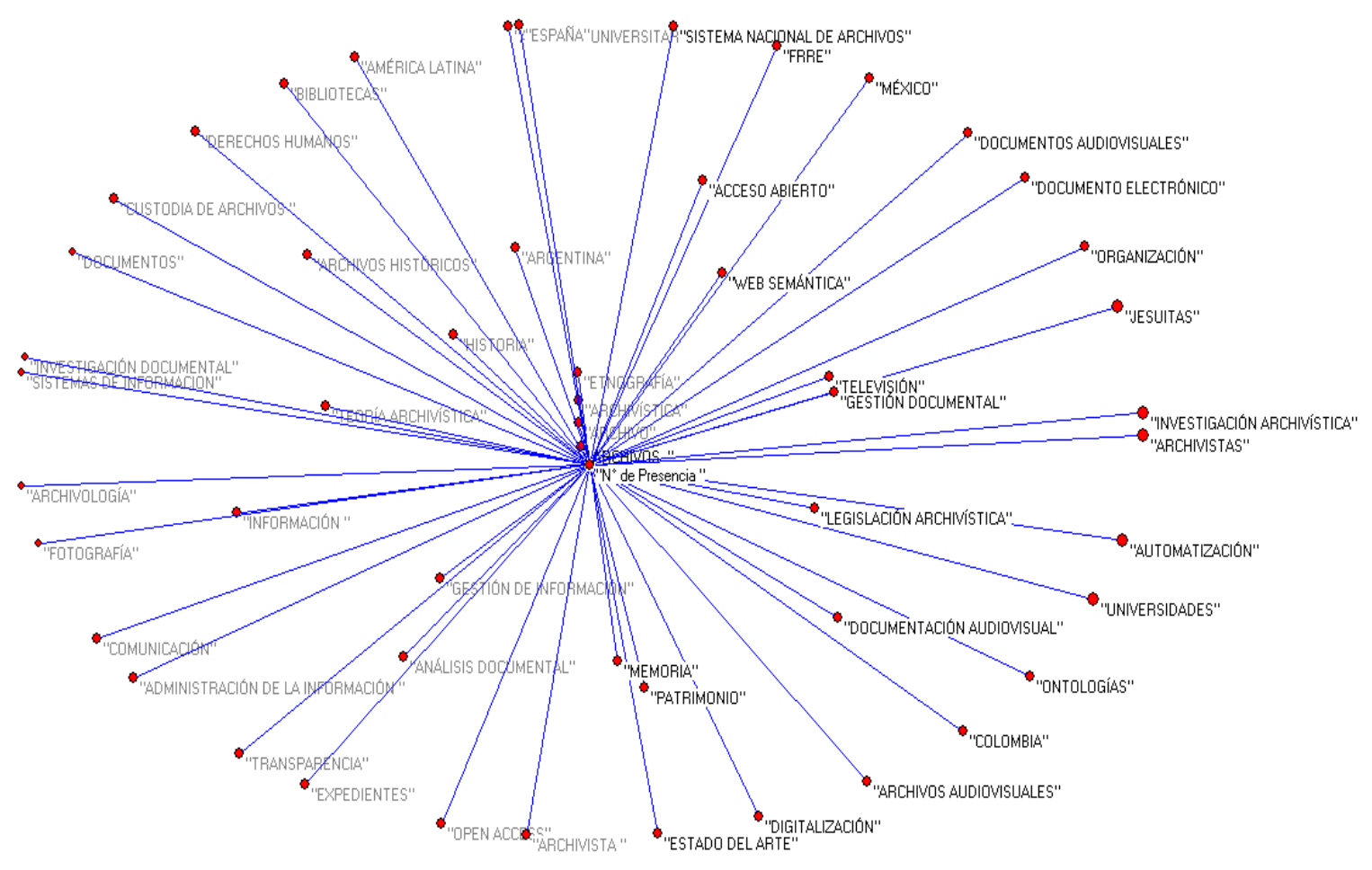

Figura 8 - Red de Palabras Temáticas más Frecuentes

En cuanto a la productividad institucional, la tabla $N^{\circ} 3$ muestra 11 de las 91 Instituciones con mayor productividad científica Archivística en Redalyc de acuerdo a las instituciones donde se desempeñan los autores, entre ellas; la primera que figura es la Universidad de la Salle con un total de 6 artículos publicados, luego le sigue la Universidad de Extremadura con 5 artículos, en tercer lugar se encuentran la Escuela de Historia de la Universidad de Costa Rica, la Universidad Carlos III, Universidad Complutense de Madrid, la Universidad del Rosario y la Universidad del Zulia con 4 publicaciones cada una, con 3 publicaciones se encuentran el Centro de Investigaciones y Estudios Superiores en Antropología Social, la Universidad de Antioquia, la Universidad de León y la Universidad de Murcia, con 2 publicaciones se encuentran un total de 10 instituciones, con una sola publicación 75 instituciones y finalmente 7 publicaciones por 9 investigadores no perteneciente a ningún tipo de institución.

\begin{tabular}{|c|c|}
\hline \multicolumn{2}{|l|}{ Productividad Institucional } \\
\hline Institución & $\mathrm{N}^{\circ}$ de Publicaciones \\
\hline Universidad de la Salle & 6 \\
\hline Universidad de Extremadura & 5 \\
\hline Escuela de Historia de la Universidad de Costa Rica & 4 \\
\hline Universidad Carlos III & 4 \\
\hline Universidad Complutense de Madrid & 4 \\
\hline Universidad del Rosario & 4 \\
\hline Universidad del Zulia & 4 \\
\hline Centro de Investigaciones y Estudios Superiores en Antropología Social & 3 \\
\hline Universidad de Antioquia & 3 \\
\hline Universidad de León & 3 \\
\hline Universidad de Murcia & 3 \\
\hline
\end{tabular}

Tabla 3 - Instituciones con mayor productividad en Redalyc 2001-2011 


\section{Conclusiones}

En base al análisis bibliométrico sobre la producción científica archivística realizada en Redalyc en el periodo 2001 2011, muestra que existe un número significativo de artículos disponibles y al servicio de los investigadores.

Uno de los dos autores de mayor productividad es Rodríguez B. Blanca. Sin embargo, la institución a la que pertenece (ULE, Universidad de León), no mantiene un nivel de alta productividad en comparación con otras instituciones.

Se registro un número de publicaciones con mayor frecuencia en la producción temática referente a los archivos, es decir como acceso a la información y como fuentes documentales, siendo lo de menor frecuencia las publicaciones sobre herramientas y procesos para la organización de los fondo documentales.

Se evidencia el crecimiento gradual en los últimos años en la productividad científica archivística de acuerdo a los indicadores de distribución por año, producción autoral, producción por país e instituciones, mostrándose prometedor el número de publicaciones futuras.

De las cincuenta revistas estudiadas, indistintamente de los indicadores de productividad obtenida, se pueden establecer como una fortaleza para consolidar la producción científica archivística, afianzamiento de las revistas como institución editorial y los autores un mayor estatus dentro de la comunidad discursiva archivística.

La productividad idiomática en español, prevalece como lengua dominante ante el inglés y el portugués.

Los indicadores de colaboración autoral en esta investigación no son los más favorables teniendo en cuenta que la mayoría de las publicaciones son producidas por un solo autor, lo que deja ver el poco compromiso de trabajo en colaboración autoral e institucional.

\section{Bibliografía}

Arias, F. (2006). El proyecto de investigación: introducción a la metodología científica. (5ª ed.). Caracas: Episteme.

Chaviano, O. (2004). Algunas consideraciones teórico-conceptuales sobre las disciplinas métricas. Acimed. Recuperado el 12 de junio de 2012 de: http://bvs.sld.cu/revistas/aci/vol12 5 04/aci07504.htm

Cruz, J. (1993) Manual de Archivística. Madrid: Pirámide.

Dictionary of Archival Terminology, 2da. Edición (1988) op. cit., n. 32.

Dorado, Y. y Martínez, A. (2010) Representatividad de las fuentes de información sobre Archivística en las Bibliotecas Especializadas en la Ciudad de la Habana. Anales. Recuperado el 4 de mayo de 2012 de: http://anales.bnjm.cu/bundles/anales/dossiers/2010/yanara dorado.pdf

Fuster, F. (2001). Archivística, archivo, documento de archivo.... Necesidad de Clarificar los Conceptos. Recuperado el 30 de mayo de 2012 de: http://revistas.um.es/analesdoc/article/view/2631/2611

Gorbea, S. (2005). Modelo teórico para el estudio métrico de la información documental. España: Ediciones TREA.

Heredia, A. (1991) Archivística general: teoría y práctica. 5 ed. Sevilla: Excma. Diputación de Sevilla.

Rubio, M. (1999). Bibliometría y ciencias sociales. Recuperado el 8 de junio de 2012, del sitio Web Proyecto Clio: http://clio.rediris.es/articulos/bibliometria.htm

Spinak, E. (1998). Indicadores cienciométricos. Ciencia de la Información. [Revista en línea], 27(2). Recuperado el 6 de junio de 2012 de: http://www.cielo.br/pdf/ci/v27n2/2729806.pdf

Tague-Sutcliffe, J. (1994). Introducción a la Informetría. ACIMED. Recuperado el 6 de junio de 2012 de: http://bvs.sld.cu/scielo.php?pid=S102494351994000300005\&scrip=sci arttext 


\section{Datos del autor}

Leomar José Montilla Peña

Licenciado en Bibliotecología y Ciencia de la Información. Egresado de la Universidad de la Habana, Facultad de Comunicación, La Habana, Cuba. Encargado de la Biblioteca Especializada del Instituto Nacional de Investigaciones Agrícola, Unidad Ejecutora Lara, Venezuela. Profesor Instructor del Programa de Formación en Ciencias de la Información de la Universidad Politécnica Territorial Andrés Eloy Blanco, Barquisimeto, Venezuela. Integrante del Comité Interinstitucional para el Fortalecimiento de la Gestión del Programa Nacional de Formación en Ciencias de la Información, Ministerio del Poder Popular para la Educación Universitaria, Caracas, Venezuela.

leomonp@gmail.com

$\begin{array}{ll}\text { Recibido-Received } & : 2012-09-17 \\ \text { Aceptado-Accepted } & : 2012-12-30\end{array}$

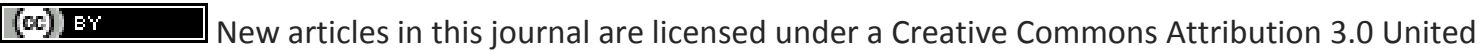
States License.

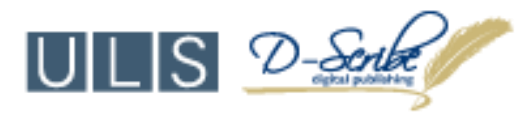

This journal is published by the University Library System of the University of Pittsburgh as part of its D-Scribe Digital Publishing Program and is cosponsored by the University of Pittsburgh Press. 